\title{
Editorial for the special issue on innovative design and applications (selected papers from ICFDM2016)
}

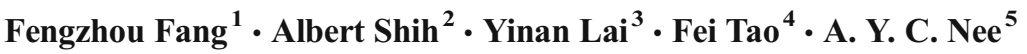

Published online: 7 November 2017

(C) Springer-Verlag London Ltd. 2017

The International Conference on Frontiers of Design and Manufacturing (ICFDM) is held biennially by the National Natural Science Foundation of China (NSFC) and ShienMing Wu Foundation of USA. It has over 20 years of history. Since1994, ICFDM has become an important platform to showcase outstanding research achievements in design and manufacturing science. Besides, the conference also aims at promoting and strengthening international academic cooperation and communication. ICFDM2016 was held in Shenyang, China, during August 10-12, 2016. The conference covers research topics on the frontiers of mechanical design and manufacturing.

Under the support of related worldwide researchers, 367 papers have been received for ICFDM2016. Each paper was reviewed by at least four reviewers, including two anonymous reviewers and two relevant session chairs. Based on the review results and recommendation from the chairs, 83 papers had been selected for oral presentations at ICFDM2016. After the second round review, 20 papers had been selected and recommended for submission to this special issue on the International Journal of Advanced Manufacturing Technology (IJAMT). In addition, 19 regular manuscripts are submitted to this special issue.

Final accepted 25 papers were based on further reviewing these 39 submissions came from over 30 universities and research institutions in 10 countries.

These accepted papers cover wide topics in the field of innovative design and applications, such as product-services system; cross-wedge rolling process for drive shaft production, quality management for fully automated manufacturing systems; product innovative method based on the criterion and noise function of robust design; comprehensive optimum and adaptable design methodology for the working mechanism of a wheel loader; friction behaviors of linear rolling bearings
Fengzhou Fang

fzfang@tju.edu.cn

Albert Shih

shiha@umich.edu

Yinan Lai

laiyn@nsfc.gov.cn

Fei Tao

ftao@buaa.edu.cn

A. Y. C. Nee

mpeneeyc@nus.edu.sg
College of Precision Instruments and Opto-Electronics Engineering, Tianjin University, Tianjin 300072, China

2 Department of Mechanical Engineering, University of Michigan, Ann Arbor, MI, USA

3 Mechanical Engineering Division, Department of Engineering and Materials Sciences, National Natural Science Foundation of China, Beijing 100085, China

4 School of Automation Science and Electrical Engineering, Beihang University, Beijing 100191, China

5 Department of Mechanical Engineering, National University of Singapore, Singapore, Singapore 
and thermal induced preload of ball bearing with temperature compensation; and interaction analysis of axial vibration and lateral vibration.

The next ICFDM will be held in Jinan, China in 2018. Researches worldwide are kindly requested to take note of ICFDM meetings in future and are most welcome to take part in ICFDM2018.

This special issue has been made possible by the strong support of Prof. A.Y. C. Nee, the Editor-in-Chief of the journal, and the joint effort of the staff of the Editorial Office. We would like to express our gratitude to the members of the Editorial Board of the Journal for encouraging us to prepare this issue, the authors for their contributions, the referees for ensuring the quality of the accepted papers, and the staff of the Springer Editorial Office and Production Department for their help in finalizing this special issue. 